\title{
A liquid level, temperature and flow control system based on
}

\section{Profibus-DP fieldbus}

\author{
Xiaojun Ding ${ }^{1,}$,, Yi Yang ${ }^{1, b}$, Shuyang Wang ${ }^{1, c}$, Yuanze Ma ${ }^{1, d}$ \\ ${ }^{1}$ The North University for Ethnics, Yinchuan Ningxia 750021, China \\ adxjabc888@163.com,, yy9678@163.com, cwangshuyang@139.com
}

\begin{abstract}
Keywords: Switched reluctance motor; Switched reluctance driver; DSP; PWM
Abstract. This paper introduced the improvement of the original control system of process control laboratory. The development of the liquid level, temperature and flow time-sharing control experiment platform adopting Profibus-DP fieldbus technology of Siemens. The newly designed control system using S7-300PLC as the master control unit, S7-224 as the slave control unit, PC and touch screen as the upper computer is able to achieve real-time monitoring on-the-spot operation, adjust control parameters, centralized management and provide convenience for experimenters. The practical operating results show that the platform not only extends the function of equipment and improves the utilization rate of equipment, but also help students understand and master the advanced control technologies, such as the technology application of PLC and touch screen, the process control engineering and the research of control algorithms.
\end{abstract}

\section{Introduction}

A factory produced a process control experimental teaching platform which can control the water tank level, boiler temperature and pipe flow, respectively. This teaching platform meets the requirements of daily teaching tasks while the equipment utilization rate is low and the degree of students benefits are limited. In order to fully mobilize the enthusiasm and creativity of students and improve the quality of teaching experiment, Siemens' advanced equipment has been used for the original single-variable control system improvement. And a control system based on Profibus-DP fieldbus technology has been proposed.

As a high-speed data transmission protocol in field-level, Profibus-DP solved the communication task in high-speed serial bus and distributed field devices in automatic control system (PLC, PC, etc.) [1]. In this paper, we selected the Siemens S7-315 PLC as the main controller (main station), PC and touch screen as the upper computer, coordinating and controlling the work order of each single-variable system that use S7-224PLC as the master controller (slave station). The system simulated the actual factory assembly line in production process, expanded the appliances' capabilities and achieved a satisfactory effect.

\section{Experimental device introduction}

The process control system experimental device flow chart is shown in Fig. 1. The controlled objects of the experimental system contains the water tank level, the boiler (jacket and liner) temperature and pipeline flow of which the upper water tank and the lower water tank form a second-order control system which can be changed into a first-order control system by closing the valve NO.6 or valve NO.7. The double-tank water level control system was composed of the cistern, the single-phase pump M101, the piezometer P1, the electric control valve FV101, the upper water 
tank, the lower water tank and so on. In addition, the boiler jacket and boiler liner form a first-order cascade control system which can be changed into a first-order control system by closing the valve NO.10. After water injection, the temperature control system could normal operation which including the single-phase pump M101, the piezometer P1, the boiler jacket and boiler liner, PT100, electric heating pipe TZ101 etc. Besides, the pipe form a first-order control system, i.e., flow control system, which contains the cistern, the single-phase pump M101, the piezometer P1, electromagnetic flowmeter FE101, the electric control valve FV101 and so on. In short, the system can complete the tasks of teaching such as first-order \& second-order single-loop control systems, and cascade control systems like liquid \& liquid, liquid \& flow and boiler jacket \& boiler interior, and the control quality research etc.

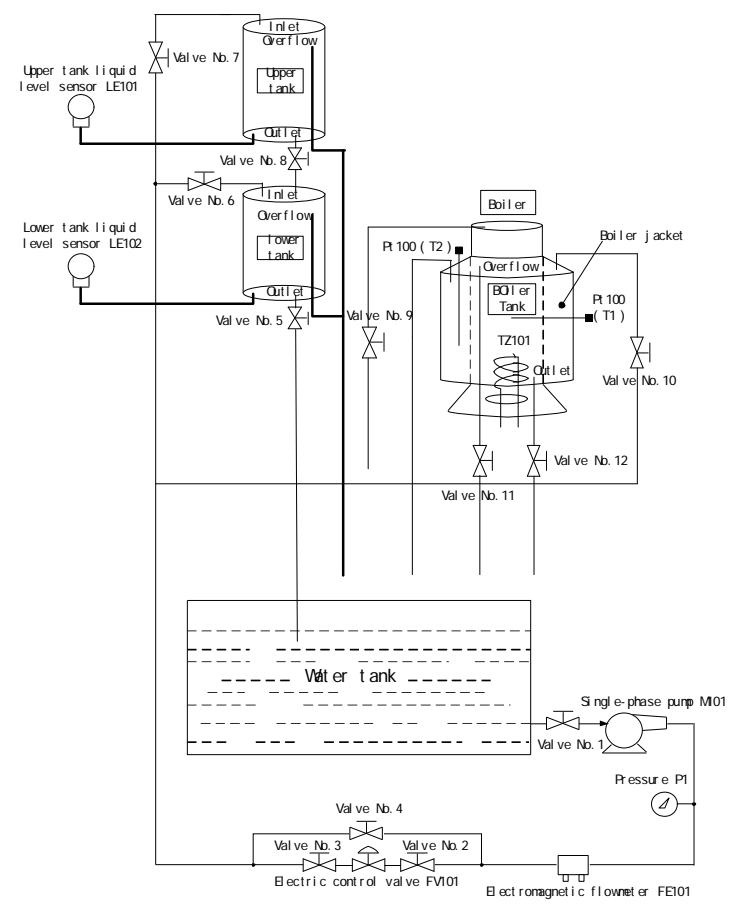

Fig.1 Experiment process flow diagram

\section{System components and network communications}

For the current mainstream industrial field bus technology, this paper designed and implemented a control system based on Profibus-DP fieldbus which composed of three parts: field devices layer, field-control layer and management and monitoring layers.

The structure of the system is shown in Fig.2. The field devices layer mainly consists of sensors and actuators. However, the field-signal input device includes a pressure sensor, a temperature sensor (Pt100) and a flow sensor (electromagnetic flowmeter) while the field-signal output device includes an electric control valve and electric heating pipe etc[2]. With input and output devices as the slave station's I/O points of Profibus-DP fieldbus, connect to the fieldbus. According to the design requirements, to achieve liquid level, temperature and flow control needs three comprehensive experimental apparatus, i.e. three slaves. From left to right in the field control layer the slaves are, in order, liquid level regulating station, temperature regulating station and flow regulating station are shown in Fig. 2. Among them, with solid lines connected devices in the scene equipment level are the actual ones to access the correspond station.

Field control layer were composed of all S7-224PLC distributed in the field. A Profibus fieldbus network is constitute by using a shielded twisted pair wire that connected from the main station to 
each slave in turn via the respective of DP port of EM277 communication module. In this paper we select S7-315PLC as the master cpu to connect with PROFIBUS fieldbus network via MPI. Each number of slave station set by hardware configuration. Each slave station's EM277 module received the output data from the master and send to the corresponding slave, and received the output data from each slave in the field and send to the master, and then to complete the data interaction between master-slave stations [3].

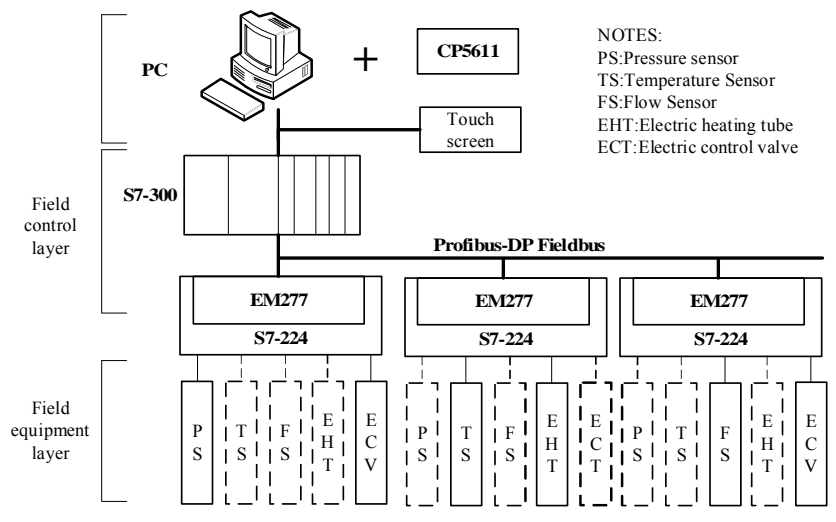

Fig.2 Process control system network architecture

The management control layer is composed of a PC and touchscreen. The host computer (PC) with a dedicated communication card CP5611 connected with PLC via MPI. The control program of each slave station is programmed by the software of STEP 7 MicroWIN SP6 V4.0, and then download to the appropriate S7-224PLC which can complete control each slave station independently. In order to accomplish the purposes of coordinating every slave station's work, the master control program should download to the S7-3C00PLC by SIMATIC Step7 [4].The touchscreen mainly monitors the system's every running status in real time, and modifies the control parameters, alarm display and historical data storage.

\section{System hardware selection}

The improved system added a S7-300PLC (master controller), a touch screen and a PC with a CP5611 communication card on the basis of the original experimental device, (controller S7-224PLC) for students to understand the pipeline operations during the actual industrial production. The major hardware selection describes as follows.

S7-300PLC selection. As a sales champion, SIMATIC S7-300 controller has been widely used in various fields of automation. It is a modular mini PLC system which contains rich CPU modules, powerful communication capabilities and to meets the medium and small range of performance requirements. The design selected model CPU315-2DP which in distributed architecture of the compact CPU module with PROFIBUS master \& slave interfaces. However, considering the actual input \& output points, easy communication and easy extension, the IO module is configures to DI8 / DO8x24V / 0.5A, the power module selects 6ES7307-1EA01-0AA0 (5A) and the communication module selects 6ES7340-1AH02-0AE0 CP340 (RS232).

Touch screen selection. The touch screen is introduced as the human-machine interface has been widely used in all aspects of industrial automation, especially in the field of metallurgy, mining, papermaking and other harsh environmental conditions. Considering the application and design costs, the design chooses Kinco model MT4523T touch screen as the user interface which owns high-speed, low-power embedded RTSC CPU, 65536 colors display, high-speed USB interface 
support and 10.4 inches TFT, easy to operate and very reliable. These characteristics meet the design requirements.

Communication module selection. The communication module mainly realizes the communication between PLC, PLC and PC, as well as other smart devices. In this design, EM277 communication module implements the master S7-300 PLC and slave S7-224PLC communication by using the communication cable to connect each station DP port in turn from the start of the master. Those connections constitute the Profibus fieldbus networks. The master S7 -300 PLC to PC (with a network card CP5611) communication is performed via MPI. While the communication between the master S7-300 PLC and touch screen is performed by a communication module 6ES7340-1AH02-0AE0CP340 (RS232) and a touch screen RS232 port, which connected with communication cables.

\section{Platform application}

After screen design of touch panel, the touch screen can completes PC monitoring configuration and human interaction. The touch screen image design uses Kinco HMIware configuration editor software which is a function packages for touchscreen screen design via Kinco application PC. The software can realize the functions of touch screen image create, switch, trend charts and alarm display etc. It is also a prerequisite software for MT5000 \& MT4000 series Kinco touchscreen screen design. The teaching experiment system's main interface development is shown in Fig. 3 by using the Kinco HMIware. The main interface screen includes two parts, flow chart of a process test configuration and experimental content. Nevertheless, the experiments includes single mode and online mode, the students switch model by clicking a button. By default in single mode. Furthermore, in stand-alone mode, the system allows six experiments to be done such as the upper tank level PID tuning, the boiler interior temperature PID tuning, electromagnetic flowmeter flow PID tuning and so on, is shown in Fig. 3.

In online model, it can the time-sharing control for liquid level, temperature and flow in the given order, and simulates the industrial automated production process. The online experimental screen based on Profibus-DP fieldbus is shown in Fig. 4, which including start, stop, reset and emergency stop four common buttons and each slave monitor screen switch buttons as well as online reset state inspection buttons. For convenience of explanation, the design of the online experiments arranged in order of level adjustment first, temperature adjustment second and flow adjustment the last. Since each slave screen contain four parts, configuration screen, numerical display, PID parameter settings and real-time curve display. Taking the inner boiler water temperature PID tuning experiment as an example to illustrate the control effect of temperature adjustment station. Besides, the monitor screen and real-time curve is shown in Fig. 5. Under the selected PID parameters, the system steady-state error is 0.37 , the adjustment time is less than 1 minute. Thus, the control performance is better. 


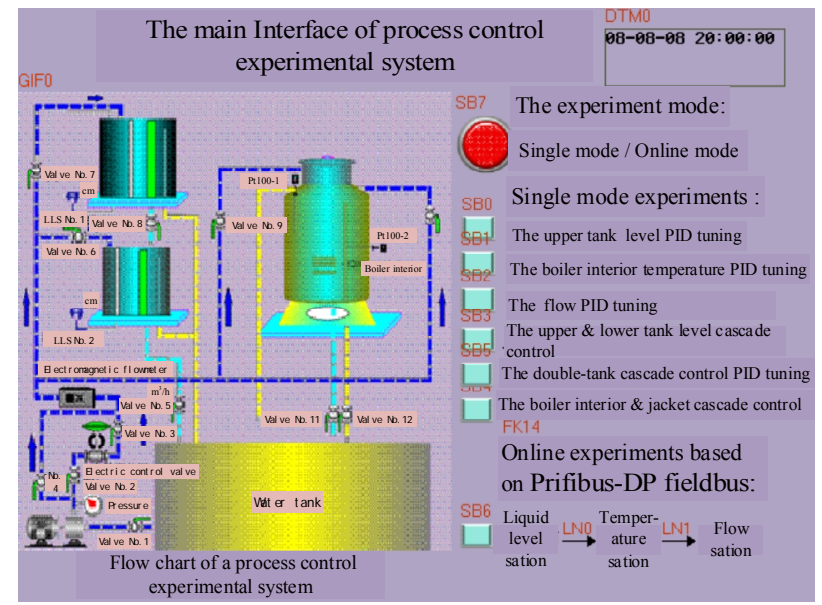

Fig.3 The teaching experiment system's main interface

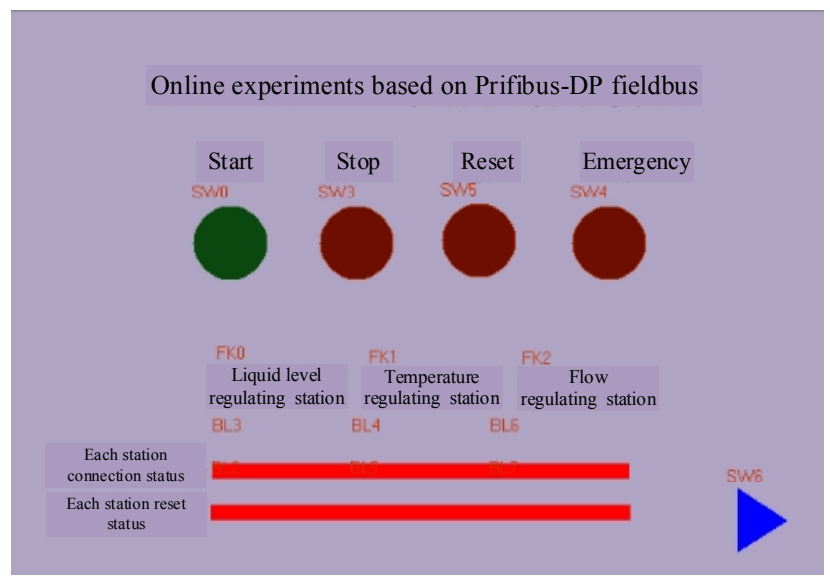

Fig.4 Online test screen based on Profibus-DP fieldbus

The designed and developed experimental platform increasing the online experiments based on Profibus-DP fieldbus, which can complete the teaching experimental of process control engineering and do experiments about communications, networking $\&$ hardware configuration. As a result, the experimental platform can help students to understand and master the structure \& the formation method based on Profibus-DP fieldbus network. In addition, it can divergent students thinking and stimulate their interests, making them to come to the fore under the severe forms of employment and enhancing competitiveness themselves.

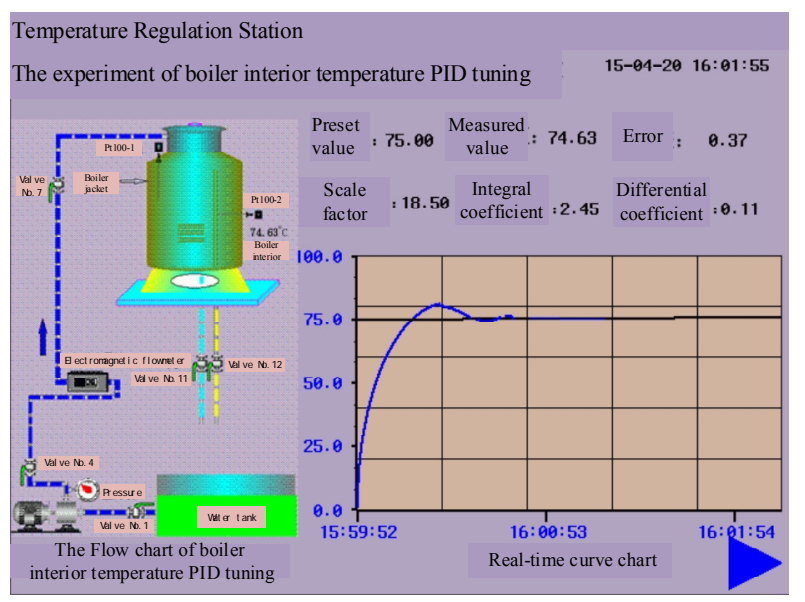

Fig.5 Temperature Regulation station monitor screen and real-time curve chart 


\section{Conclusions}

In this paper, the original system has been improved based on Prifibus-DP fieldbus technology. An unrelated single variable control system has optimized into an ordered process control system according to the order that liquid level control first, temperature control again and flow control at last. This system simulated industrial assembly line production process, and improved equipment utilization, enhanced device functionality, and to meet a higher educational requirements. The improved system provided a platform for students to learn PLC technology, touch screen technology and control the establishment method of the Prifibus-DP fieldbus. In addition, it offers technology data and support for control algorithm research and control system optimal design as well as other studies.

\section{References}

[1]Yunfeng Liang, Endian Hu, Fuchun Niu, Xiaohui Zhou, Dayong Xia. The Control System of Antibiotic Fermentation Based on PROFIBUS-DP Fieldbus [J]. Microcomputer Information, 2009, 10:61-63. (In Chinese)

[2]Xijin Gu, Liangcai Ren, Yiwen Geng, Yan Liu. Design and Implementation of PLC Experimental Platform [J]. Research and Exploration in Laboratory, 2011, 06:162-165+187. (In Chinese)

[3]Xiaojun Ding, Endian Hu, Tao Zhao. Application of PLC in Boiler Temperature Cascade Control System [J]. Instrument Technique and Sensor, 2013, 05:62-64. (In Chinese)

[4] Yu L, Wu H. The design of car transport line control system based on fieldbus technology. World Automation Congress (WAC), IEEE, pp. 1-5, 2012. 